\title{
Design of a Second Generation Electrostatic Precipitator for Martian Atmospheric Dust Mitigation of ISRU Intakes
}

\author{
James R. Phillips III, Michael R. Johansen, Jerry J. Wang, Jaysen A. Mulligan, and Carlos I. Calle \\ NASA KSC Mailcode UB-R2, Kennedy Space Center, FL 32899, USA
}

\begin{abstract}
A second generation electrostatic precipitator for use in the Martian environment has been developed by the Electrostatics and Surface Physics Laboratory (ESPL) at NASA Kennedy Space Center (KSC). This new system was designed to be modular and has three interchangeable test sections, each with a variety of replaceable high voltage electrodes, enabling optimization of the dust collection efficiency of the precipitator. It has the ability to maintain an increased atmospheric flow rate and provide more accurate dust delivery into the test section than was available in the previous prototypes. A majority of the controls for the system are provided by a software package developed to maintain a constant flow rate, low pressure, and electrode current to enable long duration performance characterization. This allows for testing of the technology in a relevant environment similar to those expected to be found in an atmospheric In-Situ Resource Utilization (ISRU) plant on Mars.
\end{abstract}

\section{Introduction}

Future surface missions to Mars involving either sample return or human exploration will encounter the challenge of successfully returning to Earth. Conventional methods rely on the spacecraft to carry the entirety of the propellant required for the return journey along on the outbound flight to Mars. Approaching the problem this way is not only much more expensive due to the increased launch mass, but also riskier for astronauts. Alternative architectures utilizing In-Situ Resource Utilization (ISRU) avoid bringing along the return propellant by converting the primarily carbon dioxide atmosphere of Mars into oxygen and methane for propellant use. This propellant is generated and stored in an ascent vehicle on the Martian surface prior to astronauts departing Earth, greatly reducing their risk of being stranded on Mars during such a mission [1]. These atmospheric ISRU plants will need to manage the challenges of low pressures (between 3 and 7 Torr) and temperatures (averaging 210 K) [2], as well as dust particles (below $10 \mu \mathrm{m}$ diameter) [3]. These particles have a constant presence in the atmosphere due to frequent dust storms and will cause both mechanical and chemical issues within the ISRU plants, so mitigation is a necessity [1].

A solution to this dust problem is the electrostatic precipitator, an active filtering technology which is widely used in industrial applications with high filtering efficiencies on Earth. It excels at removing small particles while using fewer consumables than a conventional media filter would. It can also operate at the low pressures found on Mars without limiting the gas flow into the ISRU plant to the same extent as a media filter. The precipitator prototypes under development in the Electrostatics and Surface Physics Laboratory (ESPL) at NASA Kennedy Space Center (KSC) have shown the ability to remove dust with high efficiency in a simulated Martian environment in the laboratory [4].

The electrostatic precipitator system employed by the ESPL applies a high voltage to an electrode wire installed along the axis of symmetry of a metallic cylindrical shell. This outer conductor is grounded and serves as both the dust collection electrode and the vacuum chamber in which the Martian atmosphere is simulated. The electric field, which emanates perpendicularly from the center electrode to the grounded wall of the chamber, is sufficient to generate a corona discharge within the carrier gas. This phenomenon results in charge depositing on the dust particles traveling through the precipitator. As the particles charge, they experience an increasingly repulsive electrostatic force that accelerates them away from the center electrode such that they impact and collect on the chamber wall.

Following the success of a first generation prototype, a second generation system was developed that can handle an increased atmospheric flow rate and provide more accurate dust delivery into the test section. The design of the second generation system focuses on being modular, allowing the use of various test section diameters and high voltage electrode geometries to optimize the dust collection efficiency of the precipitator. An automated software package developed for the second generation prototype can maintain a constant flow rate, low pressure, and electrode current for days at a time to conduct lifecycle and long duration performance experiments. An iterative approach was employed to develop improved dust distribution and particle counting subsystems. 


\section{Vacuum Chamber Design}

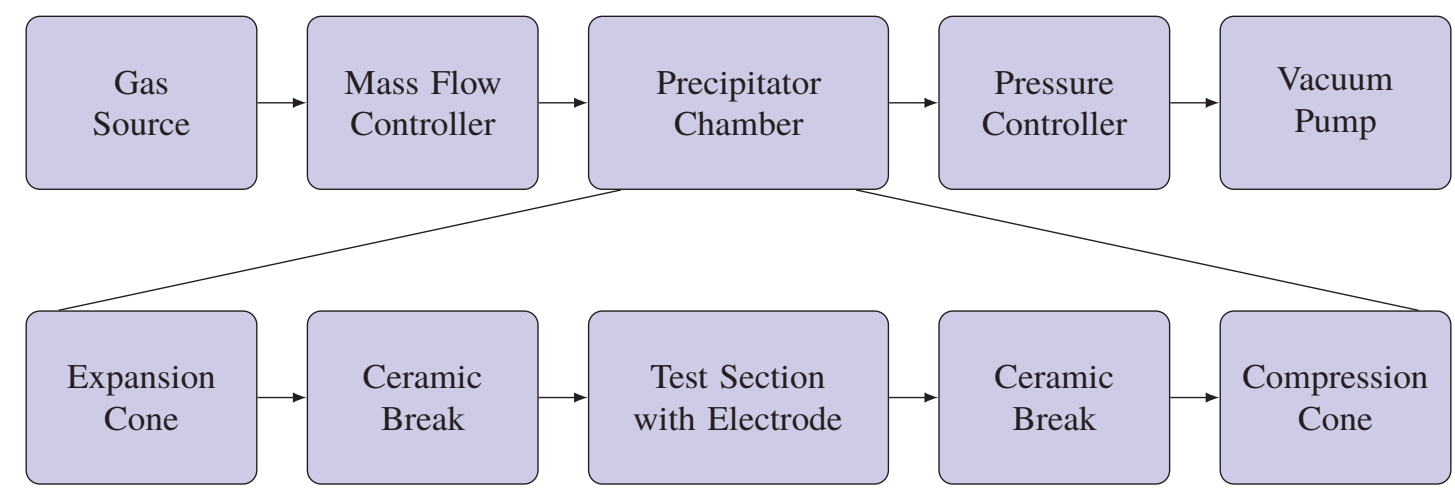

Fig. 1 Overall precipitator block diagram (top) with expanded modular test section portion (bottom)

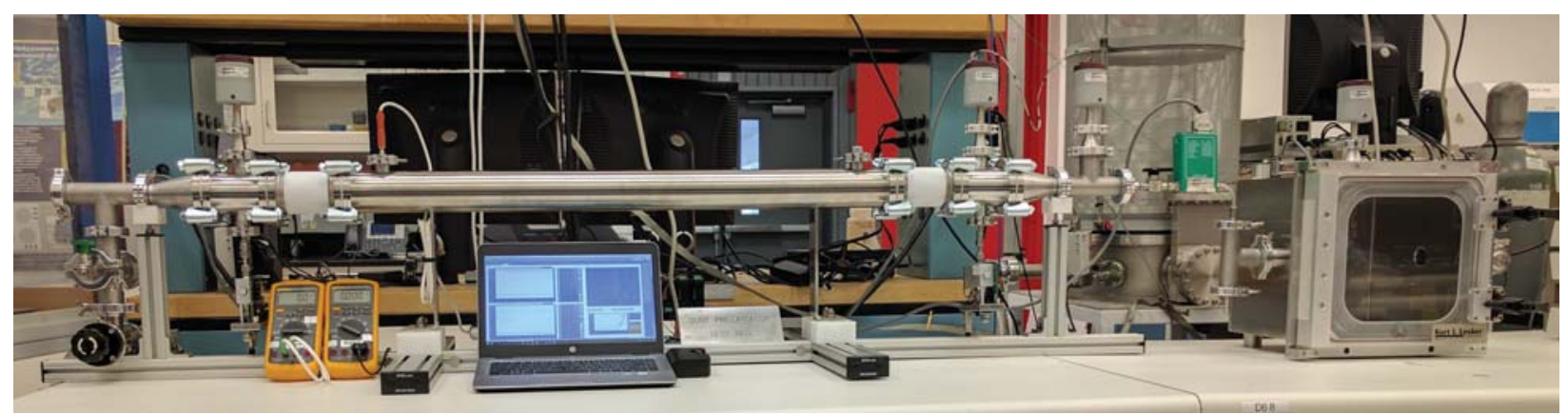

Fig. 2 Precipitator setup from right to left: gas source, dust aerosolization chamber, mass flow controller, expansion cone, upstream ceramic break, upstream particle counter and pressure transducer, precipitator chamber, downstream ceramic break, downstream particle counter and pressure transducer, compression cone, pressure controller, and vacuum pump (not shown). Computer and data acquisition electronics shown center.

The precipitator setup consists of a chamber interfaced with a gas source, atmospheric controls, and a large scroll pump that can handle continuous flow of up to 2 SLPM through the system at Martian pressures (Figure 1, top). The chamber portion was designed to be completely modular to allow easy replacement within the system to study the effects of geometry on dust collection efficiency. Each of the replaceable chambers was built around a cylindrical test section where a variety of interchangeable center electrodes could be mounted to high voltage vacuum feedthroughs. Upstream and downstream of this test section are conical adapters for interfacing the larger diameter test section to the smaller diameter permanent portion of the setup, ceramic breaks to protect the transducers from electrical discharges within the chamber, and instrumentation ports for particle count and pressure measurements (Figure 1, bottom). Three of these chambers were manufactured with diameters of approximately $7.5 \mathrm{~cm}, 10 \mathrm{~cm}$, and $15 \mathrm{~cm}$, each having an overall length of approximately $100 \mathrm{~cm}$ and an active electrode length of around $89 \mathrm{~cm}$. Figure 2 shows how the block diagram in Figure 1 was implemented in the laboratory. Atmospheric control, dust injection, and particle characterization are addressed in further detail in the following sections.

\section{Atmosphere Control}

In a previous experiment, researchers in the ESPL have shown that pure carbon dioxide behaves similarly to the Martian atmosphere under corona-generating conditions [4]. Because of this, a K-bottle of pure carbon dioxide regulated to approximately 1 bar over laboratory atmospheric pressure was used as the gas source (Figure 1, top, first block) within the precipitator chamber (Figure 1, top, center block). A scroll vacuum pump (Figure 1, top, last block) was used to evacuate the precipitator chamber to below $10^{-2}$ Torr to ensure that there would be few species other than carbon dioxide within the chamber. 
To maintain a constant flow through and pressure within the precipitator chamber, the mass flow rate both into and out of the chamber had to be controlled. When the system is in equilibrium, the mass flow sourced by the K-bottle matches that removed by the vacuum pump. To increase the pressure within the chamber, the mass flow entering the chamber must exceed that exiting the chamber, and vice versa. Mass flow controllers were installed on both sides of the chamber to achieve this behavior (Figure 1, top, blocks 2 and 4).

Controlling mass flow rate within the required range of 3 and 7 Torr rather than at the standard atmospheric pressure of 760 Torr requires a downstream flow controller to have a rated volumetric flow rate between approximately 100 and 250 times larger than that required at standard pressure. This expansion must be taken into account to enable operation of the system at the maximum standard volumetric flow rate of 2 SLPM achievable by the employed vacuum pump. While it is acceptable to use a flow controller rated for this standard volumetric flow rate to source the gas into the chamber, such a flow rate expands to upwards of 500 LPM at the lowest target pressure of 3 Torr. Since the largest orifice available in the class of flow controllers chosen to be used in the system was rated for 20 LPM at this pressure, it was necessary to install a large manual bypass valve to provide the bulk of the flow to the vacuum pump and use the flow controller for only fine adjustments to the flow. A feedback loop was established between a capacitance manometer installed on the chamber and the downstream flow controller to create a pressure controller (Figure 1, top, block 4).

\section{Dust Injection}

\section{A. In-Situ Dust Distributor}

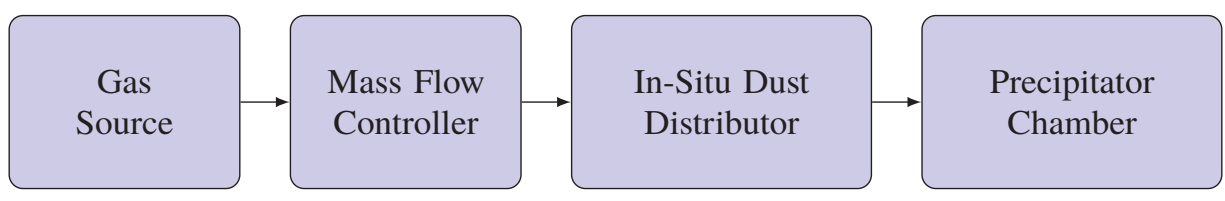

Fig. 3 In-situ dust delivery block diagram

The least complex methods of injecting dust relied on adding the dust in-situ as shown in Figure 3 . In the very first iterations of the design, a linear feedthrough with an attached cup would be filled with dust and lowered out of the flow. The cup would then be raised into the centerline of the flow where the dust was thought to be agitated and aerosolized into the precipitator. Verification showed that only a miniscule amount of the smallest diameter dust particles were being injected into the flow, so a new solution had to be found. In an attempt to inject more dust, a vibrating motor was used to sieve bulk material and drop it through a tube into the inlet of the precipitator. This approach injected too much dust too quickly, was difficult to tune, and was not able to successfully aerosolize the particles within the flow. Another problem with both of these setups was the inability to provide continuous dust delivery because the dust was contained within the low pressure side of the system. This required the chamber to be repressurized each time the dust particle reservoir needed to be replenished.

\section{B. Dust Aerosolization Chamber}

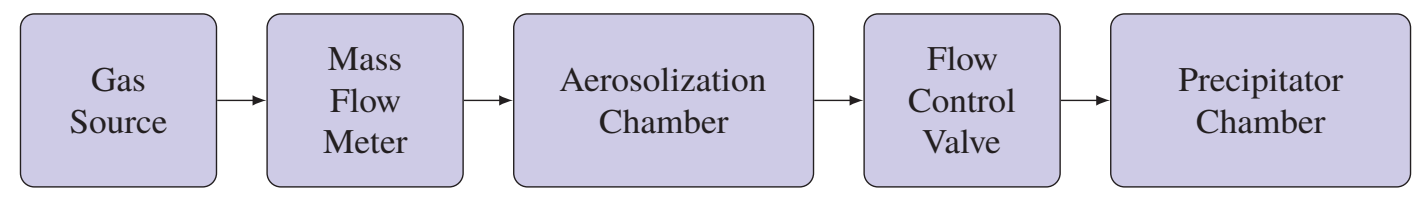

Fig. 4 Aerosolization chamber block diagram

Both of the in-situ dust delivery methods occurred on the downstream side of the mass flow controller injecting the carbon dioxide into the chamber. The difficulty in aerosolizing the dust in this manner arose from attempting to distribute it within the low pressure section of the precipitator. The low density of the gas limited the magnitude of the force applied to the dust particles and resulted in poor dispersal within the gas. This limitation was minimized by attempting to perform the dispersion in an aerosolization chamber maintained at laboratory atmospheric pressure. 
A major issue with this approach was that the mass flow controller injecting the carbon dioxide would then be directly exposed to dust which could damage the sensitive electronics within and provide false readings of the flow rate through the system. In an attempt to mitigate this problem, the mass flow controller was split into its component devices which were then monitored and controlled individually. The mass flow meter was placed upstream of the aerosolization chamber in the clean gas and the proportional flow control valve was placed downstream to deliver the dusty gas into the precipitator as shown in Figure 4.

The valve, being a purely mechanical component, was thought to be more resilient to the dust than the mass flow meter which needed to take precise measurements. Though the sensitive electronics within the flow meter were not subjected to the dusty environment, the valve remained in the section downstream of the aerosolization chamber that was constantly dusty. This caused the valve to become clogged quickly so it could not sustain the necessary dust injection volume that was required without frequent cleaning.

\section{Fluidized Dust Bed}

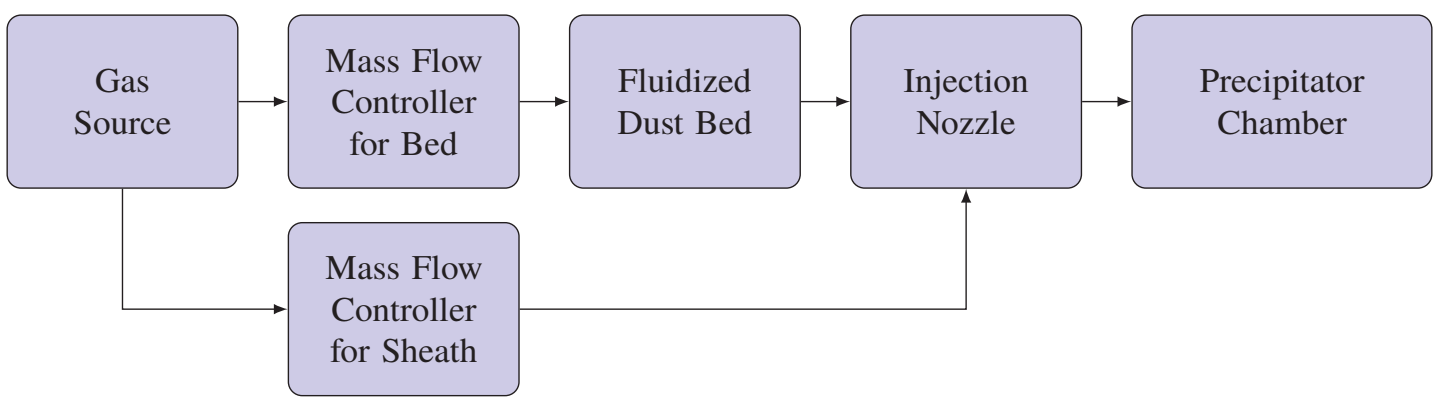

Fig. 5 Fluidized dust bed block diagram
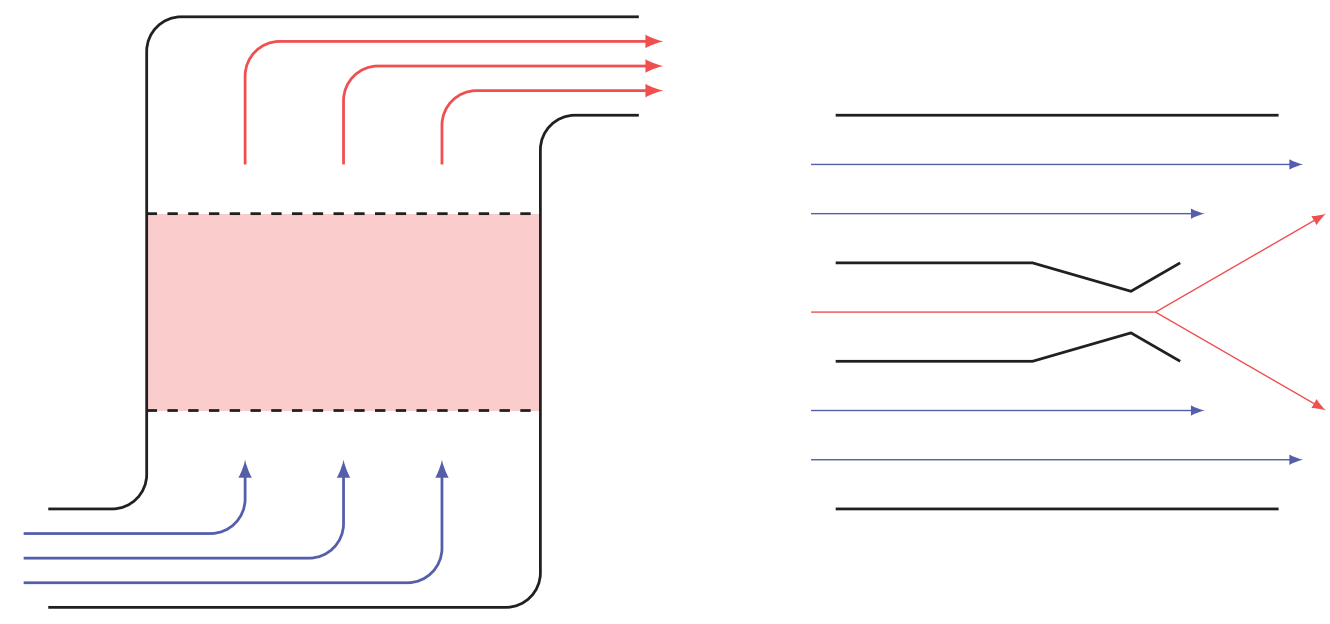

Fig. 6 Fluidized dust bed (left): Clean gas entering the bed (blue arrows from left), dust particles constrained by screens (red dotted rectangle) being entrained in the gas, and dusty gas exiting the bed (red arrows to right). Dust injection nozzle (right): Dusty gas passing through injection nozzle into downstream precipitator chamber (red arrows in center) where it is mixed with the remaining clean gas sheath flow (blue arrows around perimeter).

A fluidized dust bed (Figure 6) was constructed that utilized a porous plate that supported the dust particles from below, but allowed clean gas to pass through and agitate the particles. The bed utilized a mixture of fine particles sieved to below $10 \mu \mathrm{m}$ and larger particles sieved to below $40 \mu \mathrm{m}$. This combination of size distributions allowed the small particles to be better fluidized by incorporating larger particles in the bed. A combination of screens and baffles only allowed the smaller particles to escape the bed and be distributed into the precipitator chamber by an injection nozzle. A full cone nozzle was chosen for this purpose as it induces a swirling motion in the flow, promotes mixing of the dust into the carrier gas, and provides uniform distribution of the dust along all axes. 
The gas flow for the fluidized bed was provided via an upstream mass flow controller into the bed. This controller was configured with pressure feedback from the bed in order to maintain a constant pressure drop across the injection nozzle. The remainder of the flow required to reach the setpoint flow through the precipitator was provided by a mass flow controller throttling the sheath flow around the nozzle. This shealth flow also provided additional mixing at the tip of the injection nozzle to improve the dust dispersal. The overall setup of the system is outlined in Figure 5. A benefit to this setup is that all transducers, including the valves, are outside of the dust contaminated areas for increased reliability and longevity.

\section{Particle Categorization}

\section{A. Laser Particle Counters}

The simplest quantitative representation of the collection efficiency of a filtering device is the ratio of the number of particles passing through the filter to the total number of particles incident on the filter subtracted from unity. The first approach at obtaining this quantification was to modify standard remote laser particle counters frequently used for cleanroom atmosphere verification. This type of particle counter consists only of the laser diffraction element and associated electronics, but contains no integrated vacuum pump. These devices typically rely on a critical orifice to set a constant flow through the diffraction element, but such orifices only operate correctly when the upstream pressure is higher than the critical pressure of the orifice. Since these counters would be interfacing with a gas at the low pressures found in the Martian atmosphere, the orifice had to be removed to increase the flow rate through the counter.

While the counts returned by the counters would most likely not be accurate as they were calibrated for standard atmospheric conditions on Earth, calculation of the collection efficiency relies only on the ratio of the downstream to the upstream particle counts. Assuming that both counters were identically modified and the same inlet pressure was applied to each device, similar flow rates through the counters should be attained. It follows that errors in the counts should be roughly systematic and would cancel out in differential measurements; however, due to the small diameter of the inlet and outlet, it was difficult to cause enough flow through the diffraction element of the counters and new solutions needed to be explored.

\section{B. Physical Media Collection}
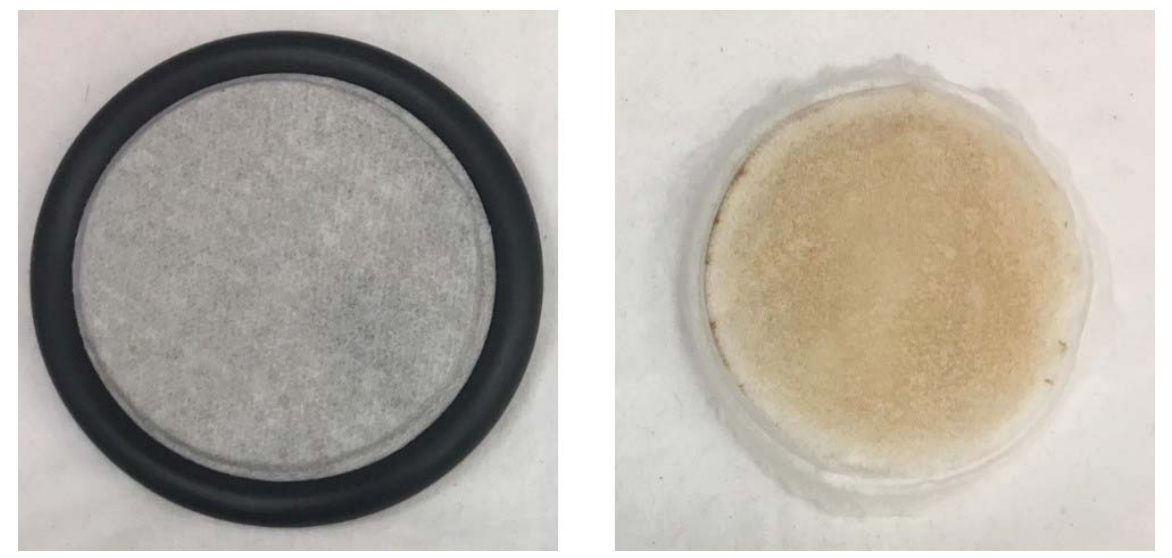

Fig. 7 Before (left) and after (right) dust distribution uniformity verification test

Since the laser particle counters were constrained to sample a single point within the flow, they were unable to provide insight into the spatial distribution of the dust. A thin, porous membrane was placed downstream of the injection nozzle to collect the entrained dust and provide visual indication of uniform distribution. Figure 7 compares the membrane before and after dust is applied and shows that the dust is evenly distributed. To better simulate conditions the system will be subjected to on the surface of Mars, the injected dust distribution must be sustainable for long durations of time. Due to the membrane becoming saturated quickly, a more comprehensive way to measure the time dependence of the dust distribution is required. Over time, the amount of dust being injected decreases and a qualitative test such as this cannot provide enough information on the decay of the dust density with time. 


\section{Laser Sheet Visualization}
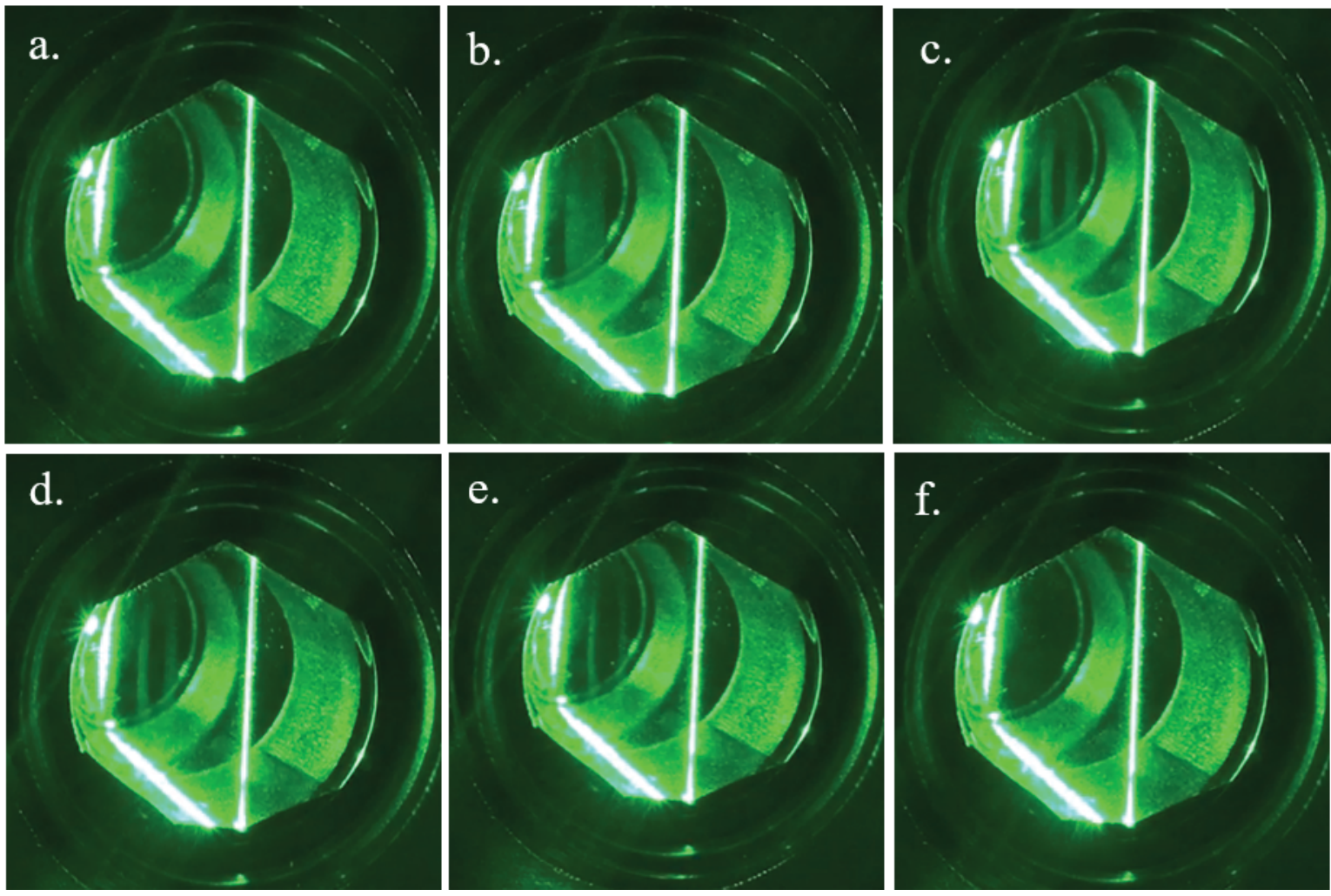

Fig. 8 Laser sheet dust visualizer in various states of precipitator operation: disabled with no dust (a), disabled with full dust entrainment (b), enabled with dust clearing (c, d, e), and enabled with maximum dust clearing (f)

Given the limited spatial measurements attainable by the laser particle counters and the tendency for the physical media to become quickly saturated, an optical approach allowing for the visualization of dust in the flow was sought. A collimated green laser was shaped through a series of lenses to produce a very thin laser sheet perpendicular to the flow within the system, illuminating the various states of operation of the precipitator for observation. Figures $8 \mathrm{a}$ and $8 \mathrm{~b}$ show the operation of the precipitator with the high voltage electrode disabled before and after the introduction of dust, respectively. The illuminated circular profile within the laser sheet in Figure $8 \mathrm{~b}$ delineates the extent of the dust entrained in the flow. Figures $8 \mathrm{c}$ through $8 \mathrm{f}$ were taken in rapid succession after the high voltage electrode was enabled. The illuminated circular profile seen within the laser sheet develops a void in the center and becomes an annulus with increasing inner radius as the dust is repelled from the high voltage electrode over time. While primarily a qualitative approach in this configuration, it is possible to modify this approach to calculate the concentration of the dust via principles of optical extinction and attain information on the temporal decay of the dust distribution.

\section{Conclusion}

A modular vacuum chamber was designed with atmospheric controls for providing constant volumetric flow rates of up to 2 SLPM at constant pressure within the range of 3 and 7 Torr. This chamber is available in several diameters and a variety of high voltage electrodes may be mounted along the centerline of the test section. The method in which dust was distributed within the chamber was iteratively improved by moving the components from inside the low pressure environment to an area external to the chamber at the higher pressure available in the laboratory.

Determining particle collection efficiency of a filtering mechanism in the low pressure environment of Mars is a difficult problem. Future work includes a more in-depth study into dust dispersion and a method to optically image individual grains. An improved method of characterizing the particle size and shape via fine particle analyzer is currently under development in the ESPL. 


\section{Acknowledgments}

- NASA Human Exploration and Operations Mission Directorate (HEOMD) Advanced Exploration Systems (AES) In-Situ Resource Utilization (ISRU) task for funding the project

- NASA Internships, Fellowships, and Scholarships (NIFS) program and NASA Pathways Intern Employment Program (IEP) for providing intern support

\section{References}

[1] Sanders, G. B., Romig, K. A., Larson, W. E., Johnson, R., Rapp, D., Johnson, K. R., Sacksteder, K., Linne, D., Curreri, P., Duke, M., Blair, B., Gertsch, L., Bouchert, D., Rice, E., Clark, L., McCullough, E., and Zubrin, R., "Results from the NASA Capability Roadmap Team for In-Situ Resource Utilization (ISRU),” 2005 International Lunar Conference, Toronto, Canada, 2005.

[2] Williams, D. R., "Mars Fact Sheet," NASA Lunar and Planetary Science, 2015. URL http://nssdc.gsfc.nasa.gov/ $\mathrm{planetary/factsheet/marsfact.html.}$

[3] Landis, G. A., "Dust obscuration of Mars solar arrays," Acta Astronautica, Vol. 38, No. 11, 1996, pp. 885 - 891.

[4] Calle, C. I., Mackey, P. J., Hogue, M. D., Johansen, M. R., Kelley, J. D., Phillips III, J. R., and Clements, J. S., “An electrostatic precipitator system for the Martian environment," Journal of Electrostatics, Vol. 71, No. 3, 2013, pp. 254 - 256. 